\title{
COMPLEJIDAD DE LA ESTRUCTURA SÍSMICA DE LA REGIÓN CENTRAL DE COSTA RICA SEGÚN UN ANÁLISIS MULTIFRACTAL
}

\author{
Mario Fernández A. ${ }^{1}$, Javier Pacheco A. ${ }^{2}$ \& Tomás Morales A. ${ }^{2}$ \\ ${ }^{1}$ Centro de Investigaciones Geofísicas (CIGEFI), Universidad de Costa Rica, \\ Red Sismológica Nacional (RSN: ICE-UCR) \\ ${ }^{2}$ Instituto de Geofísica, Universidad Nacional Autónoma de México (UNAM)
}

(Recibido 6/2/1996; Aceptado 30/9/1996)

\begin{abstract}
The Generalized dimensions (Dq) algorithm was used to investigate the fractal behavior and estimate the complexity of the spacial distribution of hypocenters in the central part of Costa Rica for the 1984-1989 and 1990-1993 periods. Results suggest that such a distribution is multifractal with a spectrum of dimensions ranging between $\mathrm{Do}=1.6$ and $D_{\infty}=0.75$. These dimensions do not diverge considerably which indicates that the seismic structure is relatively simple and homogeneous with a predominance of a spacial clustering of earthquakes instead of their propagation in the whole volume. For the $1990-1993$ period Do $=1.8$ and $D_{\infty}=1.1$ which is a consecuense of a more complex seismicity because of the Golfo de Nicoya (March 1990) and Limon (April 1991) earthquakes.
\end{abstract}

\begin{abstract}
RESUMEN: Se utilizó el algoritmo de las dimensiones generalizadas (Dq) para investigar el comportamiento fractal y estimar la complejidad de la estructura sísmica en la parte central de Costa Rica para dos períodos: 19841989, y 1990-1993. Los resultados del análisis indican que tal estructura sísmica es multifractal, con un espectro de dimensiones que varía entre $D_{\mathrm{o}}=1,6$ y $D_{\infty}=0,75$. No hay gran divergencia entre $D_{\mathrm{o}}$ y $D_{\infty}$ lo cual indica que la estructura sísmica es relativamente homogénea y simple, con predominio del agrupamiento espacial de sismos en lugar de una dispersión por todo el volumen. Para el período $1990-1993$ se calculó $D_{\mathrm{O}}=1,8$ y $D_{\infty}=1,1$, lo cual es consecuencia de una sismicidad más compleja, aquella incrementada por los terremotos del Golfo de Nicoya (marzo de 1990) y Limón (abril de 1991).
\end{abstract}

\section{INTRODUCCIÓN}

Los fractales son formas geométricas que no varían al variar la escala con que se observan y que se caracterizan por tener una dimensión no entera, un atractor y por ser tanto homogéneas como heterogéneas. En este estudio la forma o estructura por analizar es la que resulta de la distribución espacial de temblores y que se obtendrá al determinar todas las posibles distancias entre los hipocentros de la serie y compararlas con una longitud escala. La dimensión de la formas estudiadas, llamada dimensión fractal, es un parámetro que caracteriza la distribución espacial de puntos (hipocentros, en el caso de temblores), el grado de agrupamiento de los mismos (ROSSI en: KRUHL, 1994), la complejidad o irregularidad del conjunto estudiado [OKUBO \& AKI (1987), CUTLER (1993)] y el número mínimo de variables para describir el sistema [KURTHS \& HERZEL (1987), OTT (1993), CUTLER (1993), MUDELSEE \& STATTEGGER en: KRUHL (1994)]. El atractor es un subespacio del espacio en el que se encuentra la estructura 
geométrica y en el que se concentran las variables analizadas. Las estructuras fractales homogéneas se caracterizan por tener una sola dimensión mientras que las heterogéneas, llamadas multifractales, tienen todo un espectro de dimensiones fractales. Un conocimiento completo del conjunto de dimensiones del sistema es equivalente a una completa caracterización física del fractal (HENTSCHEL \& PROCACCIA, 1983). La función Dq (dimensión fractal) de una estructura sísmica contiene información sobre la concentración de la sismicidad y propiedades de agrupamiento de la misma. El análisis multifractal se considera como un método sensible para analizar la fina estructura de la sismicidad (GEILIKMAN et al., 1990) que puede utilizarse con fines predictivos [DIAO y CHAO en: KRUHL (1994), ROSSI en: KRUHL (1994), HAN \& WANG (1995)].

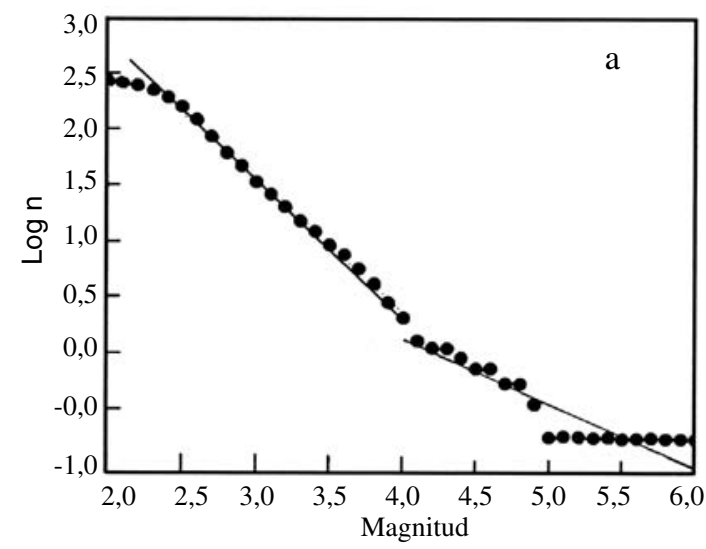

Los objetos fractales tienen gran aplicación en la Geofísica y parecen ocurrir ampliamente en el contexto de sistemas dinámicos caóticos. Procesos invariantes de escala que caen en los campos mencionados son la distribución tamaño-frecuencia de fragmentos de roca, temblores, poblaciones de fallas, erupciones volcánicas, depósitos minerales y campos petrolíferos (TURCOTTE, 1990). También ha sido demostrada la auto-similaridad en el decaimiento de réplicas asociadas a grandes temblores[n(t) $\sim \mathrm{t}-\mathrm{p}$, OMORI, 1984 en HIRATA, 1987], los procesos de ruptura cortical (FRANKEL, 1991) y en la distribución espacial de temblores $[\mathrm{N}(\mathrm{R}) \sim \mathrm{RD}$ 3, KAGAN \& KNOPOFF, 1980]. Según la fórmula anterior el número de temblores por unidad de volumen o área a una distancia $\mathrm{R}$ desde cualquier hipocentro (o epicentro) es proporcional a $\mathrm{R}^{\mathrm{D}-3}$, donde $\mathrm{D}$ es la dimensión fractal.

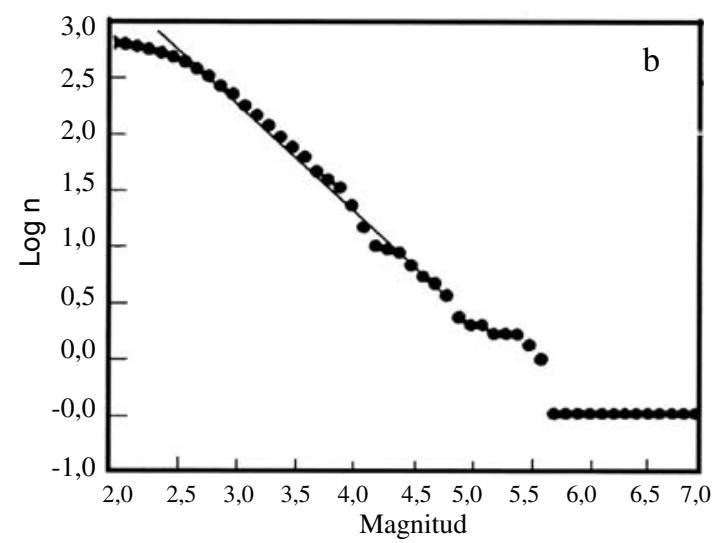

Fig. 1: Relación Frecuencia-Magnitud para la sismicidad de la zona, a) período 1984-1989, b) período 1990-1993.

Con el fin de estimar la complejidad de la estructura sísmica de la región Central de Costa Rica, se estudió la distribución espacial de los temblores, localizados por la RED SISMOLOGICA NACIONAL (RSN: ICE-UCR), en un volumen de aproximadamente $111 \times 111 \times 30 \mathrm{~km}^{3}$ para los siguientes períodos de sismicidad: 19841989 y 1990-1993. El primer período es el que mejores condiciones reune para el análisis ya que cuando se desea caracterizar fractalmente cualquier patrón, es recomendable que la variable o variables a considerar se comporten normalmente, o sea, sin cambios bruscos durante el tiempo de observación y además que la distribución Magnitud-Frecuencia no haya variado (Fig. 1); el período 1984-1989 no fue perturbado por la ocurrencia de grandes temblores y satisface los requisitos de completitud del catálogo de la microsismicidad analizada, en el rango de magnitud 2,4-4. Por el contrario, el restante período es de sismicidad incrementada tanto por el terremoto de Cóbano $(\mathrm{Mw}=7,1)$ como por el de Limón $(\mathrm{Mw}=7,7)$; a los datos de este período también se les hizo el análisis fractal para ver como cambian los resultados cuando se incluye sismicidad no característica (incrementada bruscamente). Se nota que, excepto por el enjambre de Puriscal, la distribución epicentral de los períodos 84-89 y 90-93 es muy similar (Fig. 2). 


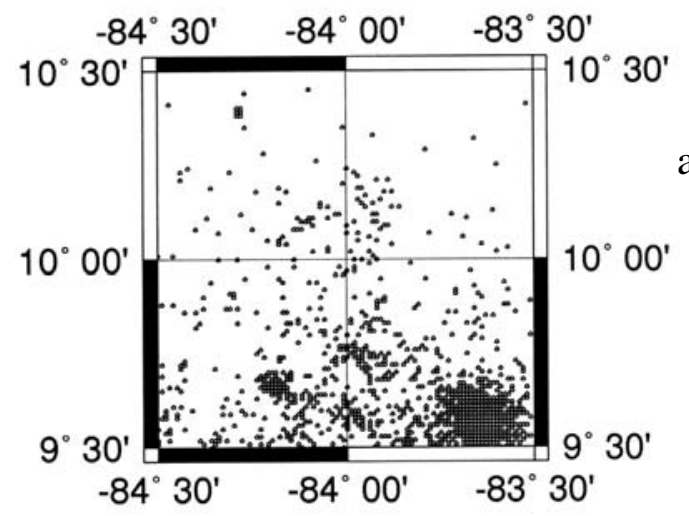

a) Sismicidad del período $1984-1989$ se incluyen 1847 sismos con profundidad menor o igual a $30 \mathrm{KM}$. Fue un período de tranquilidad sísmica en le país.

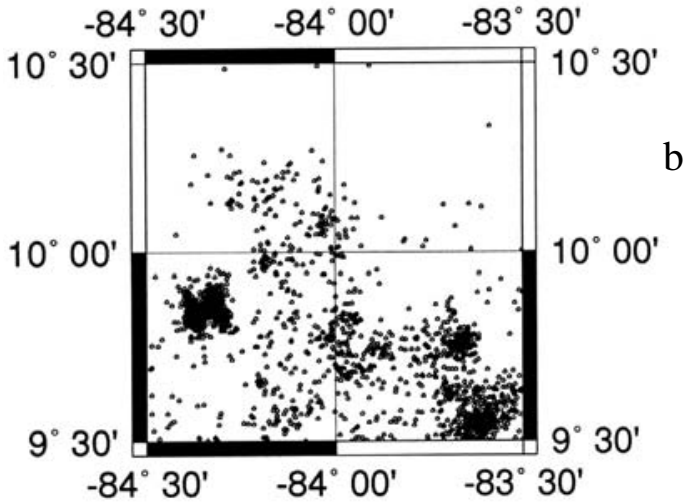

b) Sismicidad del período 1990-1993. Son 2134 eventos, se incluye la sismicidad posterior a los terremotos de Cóbano y Limón. La sismicidad al cnetro del tercer cuadrante es la de Pejibaye de Turrialba de 1993.

Fig. 2: Distribución epicentral de temblores en los períodos considerados. a)-Sismicidad del período 1984-1989. Se incluyen 1847 sismos con profundidad menor o igual a $30 \mathrm{~km}$. Fue un período de tranquilidad sísmica en el país. b)- Sismicidad del período 1990-1993. Son 2134 eventos sísmicos entre los que se incluyen aquellos posteriores a los terremotos de Cóbano y Limón. La sismicidad al centro del tercer cuadrante es la de Pejibaye de Turrialba de 1993.

\section{METODOLOGÍA}

El cálculo de las dimensiones generalizadas (GRASSBERGER, 1983; GRASSBERGER \& PROCACCIA, 1983; HENTSCHEL \& PROCACCIA, 1983) de la distribución espacial de la sismicidad del área en cuestión, se hizo con la función de densidad nj y la integral de correlación $\mathrm{C}_{\mathrm{q}}$ (KURTHS \& HERZEL, 1987; HIRATA \& IMOTO, 1991). Sus expresiones matemáticas son las siguientes:

$$
n_{j}(r)=\frac{1}{N-1} \sum_{j \neq k} \Theta\left(r-\left|x_{j}-x_{k}\right|\right),
$$

donde Q(s) es el contador llamado función Heaviside que se define como:

$$
\begin{gathered}
\Theta(S)=\left\{\begin{array}{l}
1, \text { si } s \geq 0, \\
0, \text { para otros valores }
\end{array}\right. \\
C_{q}=\left(\frac{1}{N} \sum_{j=1}^{N}\left[n_{j}(r)\right]^{q-1}\right)^{\frac{1}{q-1}} \alpha r^{D_{q}}
\end{gathered}
$$

donde $\mathrm{r}$ es la longitud escala, $\mathrm{N}$ el número total de sismos utilizados, $\mathrm{x}$ la variable dinámica o posición del hipocentro, q la variable estática del sistema y $\mathrm{D}_{\mathrm{q}}$ la dimensión fractal. Aclarando, todo sistema caótico que pueda ser descrito por una ecuación matemática incluye dos clases de variables: dinámicas y estáticas. Las primeras son las cantidades fundamentales que cambian siempre y las segundas, generalmente son parámetros. Estos parámetros son importantes porque permiten conocer que tan sensible es el sistema al cambio de las condiciones iniciales y por tanto, que tan homogéneo es. A pesar de que una estructura multifractal tiene todo un espectro de dimensiones fractales, se considera que la dimensión fractal de la misma es $\mathrm{D}_{0}$ (KURTHS \& HERZEL, 1987) la que es obtenida con el armónico $(\mathrm{q}=0)$.

La función $n_{j}$ determina la distancia entre cada pareja de temblores y compararla con una distancia $\mathrm{r}$ dada, dando un valor de nj para cada r. Esta función proporciona la distribución de temblores de acuerdo a la distancia entre ellos y por eso permite estimar propiedades de concentración y agrupamiento de los mismos. Si la distribución de temblores es auto-similar, la relación entre la Integral de Correlación Generalizada $\mathrm{Cq}(\mathrm{r})$ y la distancia $\mathrm{r}$ obedece la ecuación (2) $\mathrm{Cq}(\mathrm{r}) \sim \mathrm{r}^{\mathrm{Dq}}$ a pequeñas distancias $\mathrm{r}$. La estructura auto-similar de la distribución espacial de temblores se obtiene al graficar logarítmicamente 
$\mathrm{C}_{\mathrm{q}}(\mathrm{r})$ contra $\mathrm{r}$, aquella región donde la gráfica muestre linealidad será la región de auto-similaridad. Obviamente, si no hay linealidad la estructura no es auto-similar y no tendrá por tanto, comportamiento fractal. De las partes lineales de cada curva se obtiene las dimensiones fractales del conjunto. La divergencia de estas dimensiones refleja la heterogeneidad del atractor (KURTHS \& HERZEL, 1987) y de hecho, si no hay divergencia, el atractor sería homogéneo (para cualquier valor de q la dimensión fractal es la misma).

\section{RESULTADOS}

Se calculó la función densidad local para la distribución de temblores en la región central de Costa Rica con la ecuación (1) y luego se usaron

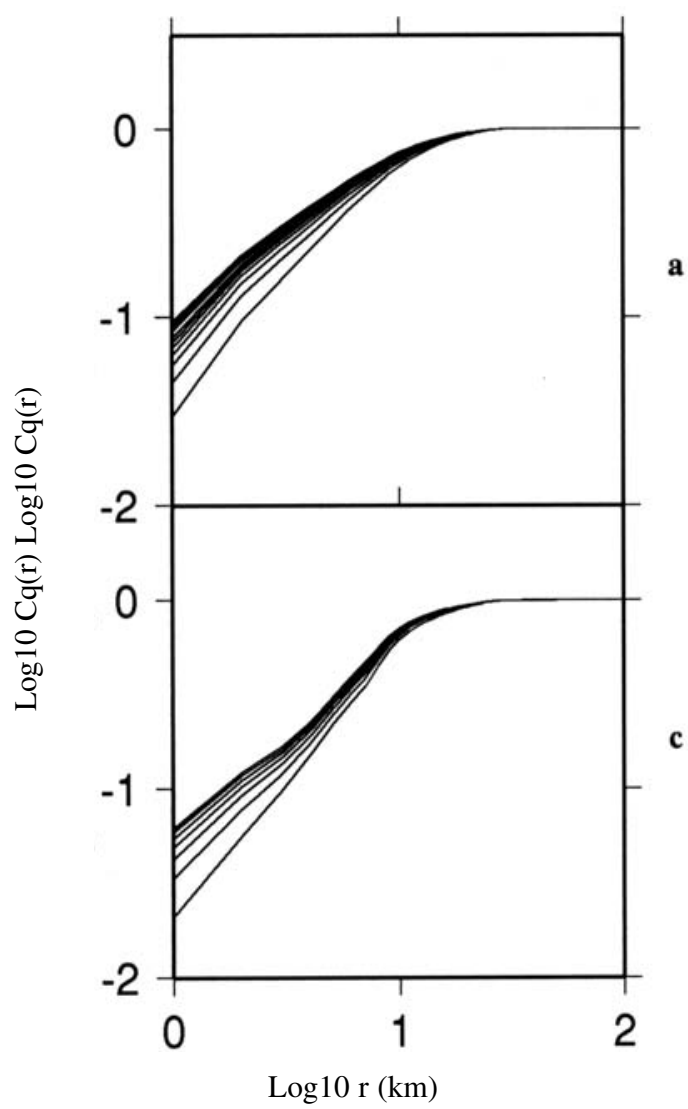

estos cálculos para obtener los valores de $\mathrm{Cq}(\mathrm{r})$. La figura 3 muestra la gráfica de $\mathrm{Cq}(\mathrm{r})$ contra $\mathrm{r}$ en escala logarítmica variando q entre 0 y 25 ; hasta 25 porque en ese valor se estabiliza $\mathrm{Dq}$. Las figuras a y c son las curvas completas; en ellas se nota una ligera flexura a una distancia variable entre 2 y $3 \mathrm{~km}$ la cual obedece al hecho de que la forma de la parte inicial de las curvas está dominada por los errores de localización a pequeñas distancias $\mathrm{r}$ [KAGAN \& KNOPOFF (1980), HIRATA \& IMOTO (1991)]. Las curvas están muy juntas, muestran linealidad, y a distancias mayores de $10 \mathrm{~km}, \mathrm{Cq}(\mathrm{r})$ se transforma en una constante. Las figuras b y d son las regiones de linealidad de las figs a, y c respectivamente; como se observa, hay auto-similaridad y un atractor en el rango de distancias de 4 a $10 \mathrm{~km}$ para las figuras $3 \mathrm{~b}$ y $3 \mathrm{~d}$.

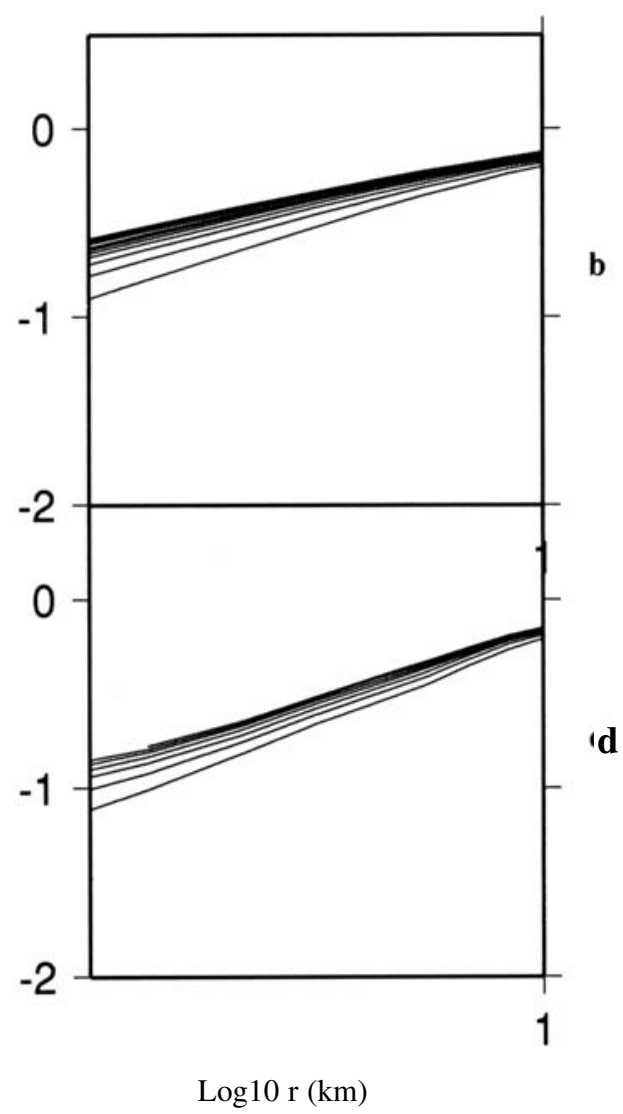

Fig. 3: Gráficos Cq contra r y su región de autosimilaridad en escala logarítmica. a)- Período 1984-1989, b)- Linealidad de las curvas 84-89, c)- Período 1990-1993, d)- Linealidad de las curvas 90-93. El rango de distancias en el que la proporcionalidad de la ecuación 2 está bien atrapada es 3-10 km. En ese rango la distribución espacial de hipocentros para la región central de Costa Rica tiene una estructura auto-similar. 
Los valores $\mathrm{D}_{\mathrm{q}}$ se obtuvieron a partir de las pendientes de las curvas de las figuras $3 \mathrm{~b}$ y $3 \mathrm{~d}$, utilizando el método de mínimos cuadrados. La figura 4 es la gráfica de $\mathrm{D}_{\mathrm{q}}$ contra q para cada uno de los períodos analizados. Se observa como Dq decae muy lentamente y se estabiliza en $q=25$ con valores de 0,75 para la sismicidad del período 1984-1989 y con 1,10 para el período 19901993. El valor de estabilización es el límite inferior de Dq y se denomina dimensión infinita. Así,

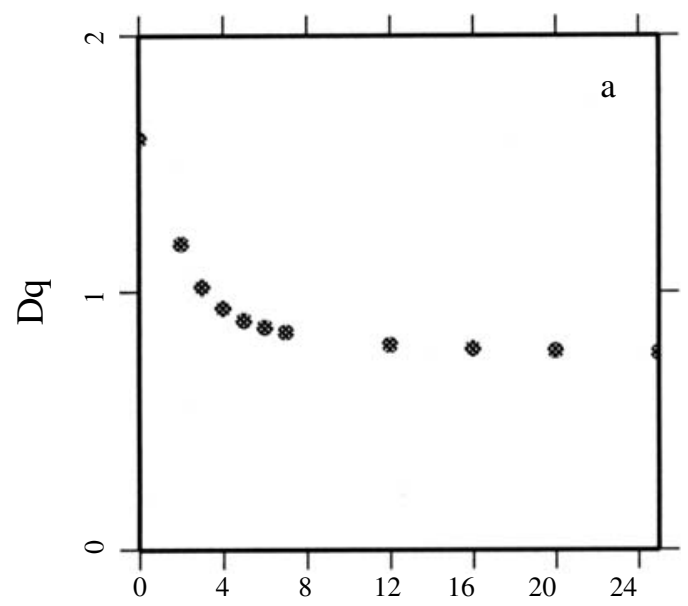

se concluye que la dimensión infinita de la estructura sísmica correspondiente al período 1984-1989 es 0,75 y la del período 1990-1993 es 1,1. La dimensión fractal de los sistemas analizados es: 1,6 para el período 1984-1989, y 1,8 para el período 1990-1993. Estos resultados indican que los sismos no están ocurriendo a lo largo de una linea pero tampoco llenan el plano del área estudiada y que las profundidades de los temblores son muy semejantes.

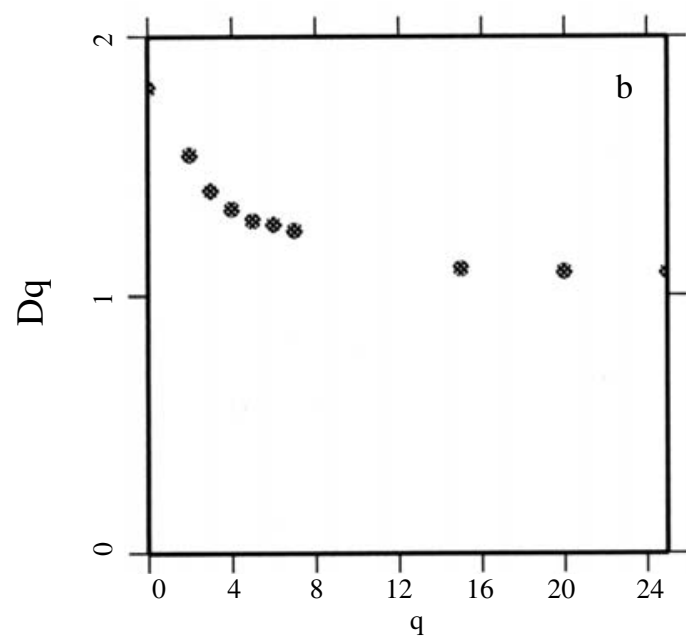

Fig. 4: Dimensiones generalizadas graficadas contra la variable estática $q$, para $q$ entre 0 y 25 . Los valores para los primeros $q$ se muestran continuamente, no asi el resto, debido a que el cálculo de $\mathrm{Cq}(\mathrm{r})$ para un $q$ y 1000 hipocentros toma aproximadamente 1 hora en tiempo de computador.

a)- Período 1984-1989. $\mathrm{D}_{\mathrm{O}}=1,6$. La estabilidad de Dq se alcanza en $q=25$ con magnitud 0,75 . Dq se obtuvo de las pendientes de la figura 3b por el método de mínimos cuadrados. b)- Período 1990-1993. $\mathrm{D}_{\mathrm{o}}=1,8$. Estabilidad en $q=25, D_{\infty}=1,1$. Dq derivado de las pendientes de la figura $3 \mathrm{~d}$.

\section{COMPARACIÓN CON OTROS ESTUDIOS Y CONCLUSIONES}

El siguiente es un listado de dimensiones fractales que han sido obtenidas para diferentes procesos:

1- $\quad$ Sistema de fallas en Japón: $1,05<\mathrm{D}<1.6$ (HIRATA, 1989)

2- Falla San Andrés: $1,0008<\mathrm{D}<1,0191$ (AVILES et al., 1987), 1,1 < D < 1,4 (OKUBO \& AKI, 1987)

3- Distribución hipocentral de réplicas en Nagano, Japón $\mathrm{D}=2,7$ [YOSHIDA \& MIKAMI (1986) en: HIRATA (1987)]

4- Distribución hipocentral en Kanto, Japón
$\mathrm{D}_{2}=2,2,>D_{\infty}=1,17$ (HIRATA \& IMOTO, 1991)

5- $\quad$ Alrededor del reservorio Koyna, India $\mathrm{D}=$ 4,4 (SRIVASTAVA et al., 1994).

En el caso de la falla San Andrés hay diferencias entre los dos estudios porque uno consideró las ramificaciones de la falla y el otro no. Obviamente que ello aumenta la complejidad del sistema y de ahí la dimensión más alta obtenida por OKUBO \& AKI (1987). Los resultados obtenidos por AVILES et al. (1987) para la traza principal de la falla les lleva a concluir que esta falla tiene un ligero pero importante comportamiento fractal y que la superficie de la falla es simple o casi planar más que áspera. El caso del reservorio 
de Koyna es muy interesante puesto que en él han ocurrido sismos de magnitud 6,5 y el período de recurrencia de temblores es progresivo en el tiempo mostrando no-linealidad. La dimensión fractal calculada para varios períodos de este sistema fue 4,4. SRIVASTAVA et al (1994) concluyen que ese sistema requiere al menos 5 parámetros para ser resuelto y que probablemente el llenado del reservorio influye en la dimensión del atractor extraño.

Los resultados obtenidos en este trabajo indican que la distribución hipocentral de la sismicidad de la región central de Costa Rica es una estructura auto-similar en el rango de distancias de 3 a $10 \mathrm{~km}$ con dimensión fractal 1,6 y dimensión infinita 0,75 , lo cual indica que predomina un intenso agrupamiento en vez de una gran dispersión de los focos. Lo anterior se verifica también observando que a más de $10 \mathrm{~km}$ el valor de la integral de correlación no varía con la distancia y esto sugiere que la gran mayoría de temblores se encuentran a distancias pequeñas unos de otros. Se cumple que $\mathrm{D}_{0}=1,6>\mathrm{D}_{2}=1,19$ $>\mathrm{D}_{3}=1,02>\ldots \ldots \ldots .>D_{\infty}=0,75$ para el período más representativo de la estructura fractal (1984-1989), lo cual muestra que la misma es heterogénea pero muy ligeramente (poca divergencia entre $\mathrm{D}_{2}$ y $D_{\infty}$ ). Por lo tanto, se considera que esta estructura sísmica no es extremadamente compleja.

Los hipocentros no se concentran linealmente pero tampoco llenan el plano del área estudiada y se agrupan en zonas permanentes de debilidad cortical, donde ocurren a profundidades muy similares. Esas zonas de debilidad podrían ser consecuencia de procesos compresivos y de movimientos epirogénicos en el área. Se determinó que la dimensión fractal de la estructura sísmica para el período 1990-1993 fue superior a la del período 1984-1989, lo cual se obedece a un incremento en la sismicidad observada no atribuible a cambios en la conformación de la red, tales como incremento del número de estaciones sísmicas y mejoramiento de la corbertura de la misma. Además, nuevas fuentes sísmicas se activaron como fueron los casos de Puriscal y Pejibaye de Turrialba, lo que transformó el sistema a uno ligeramente más complejo.

Los grandes esfuerzos generados en los bordes de las placas tectónicas son los responsables de procesos como la sismicidad y la de- formación cortical, los cuales pueden ocurrir hasta cientos y miles de $\mathrm{km}$ de los límites tectónicos (SORNETTE et al., 1993). A través de análisis de la distribución espacial de fallas (HIRATA, 1987; SORNETTE et al., 1993) y temblores (KAGAN \& KNOPOFF, 1980; HIRATA \& IMOTO, 1991) se ha demostrado que estos procesos son autosimilares, o sea, no se distribuyen al azar sino organizadamente. Esto ocurre cuando un solo factor gobierna los procesos, ya que cuando intervienen varios factores en la generación de los mismos, cada cual tratará de imprimir su huella en el espacio de estado (cada cual genera su patrón característico) con lo cual aumentará la complejidad en los sistemas afectados y hasta es posible que se pierda la autosimilaridad. AVILES et al (1987) encontraron varias tendencias en las curvas que construyeron para estudiar la geometría de la Falla de San Andrés; esto lo atribuyen a que varios factores gobiernan tal geometría. Por lo anterior, se considera que un solo factor es el que está gobernando la deformación y la sismicidad en la parte central de Costa Rica, ese sería el esfuerzo generado en la Fosa Mesoamericana. La presencia de este factor está comprobada en la deformación existente en la parte central de Costa Rica (ARIAS \& DENYER, 1991). Otro factor como sería un margen transcurrrente con características y patrones de deformación propios, no parece estar presente por cuanto no se observa otro estilo estructural (uno diferente al patrón conjugado) en la zona ni la sismicidad cubre por completo el volumen del área estudiada. Siendo que tal margen atravesaría casi por el centro de la zona, se esperaría un volumen de corteza más fracturado y lleno de sismos y por consecuencia una dimensión fractal un poco mayor que la obtenida en este estudio. Es cierto que se han activado zonas de baja sismicidad en el pasado como Puriscal y Pejibaye (el resto tiene la misma distribución en los períodos 1984-1989 y 1990-1993) y que se han formado nuevas fallas (caso de Puriscal), lo cual podría indicar la evolución de un sistema dinámico, pero aún con todo ello la complejidad de la estructura sísmica no es extrema. Como patrón dinámico de comparación, vale reflexionar sobre la situación del reservorio de Koyna donde se combinó la tectónica y un esfuerzo inducido para generar un atractor excesivamente complejo. 


\section{AGRADECIMIENTO}

Se agradece profundamente al INTERNATIONAL DEVELOPMENT RESEARCH CENTRE (IDRC) de Canadá por el soporte económico para realizar esta investigación. Asimismo, nuestro sincero agradecimiento al personal de la Oficina Regional del IDRC para América Latina y el Caribe (LACRO) y al del Instituto Panamericano de Historia y Geografía (IPGH) por su gran colaboración durante la investigación. A la RED SISMOLOGICA NACIONAL (RSN:ICEUCR) por haber suministrado los datos sismológicos para este estudio.

\section{BIBLIOGRAFÍA}

ARIAS, O. \& DENYER, P., 1991: Estructura geológica de la región comprendida en las hojas topográficas Abra, Caraigres, Candelaria y Río Grande, Costa Rica. - Rev. geol. Amer. Central, 12: 61-74.

AVILES, C., SCHOLZ, C. \& BOATWRIGHT, J., 1987: Fractal analysis applied to characteristic segments of the San Andreas Fault. - J. Geoph. Res., 92, B1: 331-344.

CUTLER, C., 1993: Review of theory and estimation of fractal dimension. - Technical Report Series, University of Waterloo, 107 págs.

FRANKEL, A., 1991: High-frequency spectral falloff of earthquakes, fractal dimension of complex rupture, $b$ value, and the scaling of strength on faults. - J. Geophys. Res. 96,B4: 6291-6302.

GEILIKMAN, M., GOLUBEVA, T. \& PISARENKO, V., 1990: Multifractal patterns of seismicity. - Earth and Planetary Science Letters, 99: 127-132.

GRASSBERGER, P., 1983: Generalized dimensions of strange attractors. - Physics Letters, 97A,6: 227-230.

GRASSBERGER, P. \& PROCACCIA, Y., 1983: Characterization of strange attractors. -
Phys. Res. Lett., 50,5: 346-349.

HAN, W. \& WANG, W., 1995: Studies of the change rates of capacity dimension and information dimension and earthquakes prediction in 11 seismic zones of SichuanYunnan area. - Fractals, 3, 1: 105-112.

HENTSCHEL, H.\& PROCACCIA, I., 1983: The infinite numbers of generalized dimensions of fractals and strange attractors. - Physica, 8D: 435-444.

HIRATA, T., 1987: Omori's power law aftershock sequences of microfracturing in rock fracture experiment. - J. Geoph. Res. 92,B7: 6215-6221.

HIRATA, T., 1989: Fractal dimension of fault system in Japan: Fractal structure in rock fracture geometry at various scales. - PAGEOPH, 131, Nos 1/2: 157-170.

HIRATA, T. \& IMOTO, M., 1991: Multifractal analysis of spacial distribution of microearthquakes in the Kanto region. Geophys J. Int. 107, 1:155-162.

KAGAN, Y. \& KNOPOFF, L.: The spacial distribution of earthquakes: The two-point correlation function. - Geophys. J. R. Astron. Soc., 62: 303-320.

KRUHL, J, 1994: Fractals and dynamics systems in geoscience. - Springer-Verlag, Berlín, 421 págs.

KURTS, J. \& HERZEL, H., 1987: An atractor in a solar times series. - Physica, 25D: 165172.

OKUBO, P. \& AKI, K., 1987: Fractal geometry in the San Andres Fault System. - J. Geoph. Res., 92, B1: 345-355.

OTT, E, 1993: Chaos in dynamical systems. Cambridge University Press, 385 págs.

SRIVASTAVA, H., BHATTACHARYA, S. \& SINHA, K., 1994: Strange attractor dimension as new measure of seismotectonics around Koyna reservoir, India. - Earth and 
Planet Science Letters 124: 57-62.

SORNETTE, A., DAVY, P. \& SORNETTE, D., 1993: Fault growth in brittle-ductile experiments and the mechanics of continental collisions. - J. Geophys. Res. 98, B7: 12,
111-12, 139.

TURCOTTE, D., 1990: Fractals, Chaos, self-organized criticality and tectonics. - Terra Nova, 4: 4-12. 